\title{
Ampliação do limite norte de distribuição geográfica de Catathyridium garmani (Jordan \& Goss 1889) (Actinopterygii: Achiridae)
}

\author{
Alexandre Clistenes de Alcântara Santos ${ }^{1,2}$, Jailza Tavares Oliveira Silva ${ }^{1}$, \\ Perimar Espírito Santo de Moura ${ }^{1}$ \& Marconi Porto Sena ${ }^{1}$ \\ ${ }^{1}$ Laboratório de Ictiologia, Universidade Estadual de Feira de Santana - UEFS, \\ Av. Transnordestina, s/n, CEP 44036-900, Feira de Santana, BA, Brazil \\ ${ }^{2}$ Autor para correspondência: Alexandre Clistenes de Alcântara Santos, \\ e-mail: alexandreclistenes@gmail.com
}

SANTOS, A.C.A, SILVA, J.T.O., MOURA, P.E.S., \& SENA, M.P. Geographic distribution ampliation of Northern limit of Catathyridium garmani (Jordan \& Goss 1889) (Actinopterygii: Achiridae). Biota Neotrop., 9(1): http://www.biotaneotropica.org.br/v9n1/en/abstract?short-communication+bn02409012009.

Abstract: Catathyridium garmani (Jordan \& Goss, 1889) is being recorded for the first time in Northeastern Brazil on the basis of twenty-six specimens collected in the Paraguaçu river estuary, São Félix municipality, Bahia state. This note relates the septentrional geographic distribution's ampliation of this species, which was restricted to Rio de Janeiro state. Meristic and morphometric data from the collected species are presented. Keywords: occurrence, morphometric, Bahia.

SANTOS, A.C.A, SILVA, J.T.O., MOURA, P.E.S., \& SENA, M.P. Ampliação do limite norte de distribuição geográfica de Catathyridium garmani (Jordan \& Goss 1889) (Actinopterygii: Achiridae). Biota Neotrop., 9(1): http://www.biotaneotropica.org.br/v9n1/pt/abstract?short-communication+bn02409012009.

Resumo: Catathyridium garmani (Jordan \& Goss, 1889) é registrada pela primeira vez para o litoral nordeste do Brasil com base em 26 exemplares coletados no estuário do rio Paraguaçu, município de São Félix, estado da Bahia. Amplia-se desta forma o limite setentrional da espécie que até o momento restringia-se ao estado do Rio de Janeiro. São ainda apresentados dados morfométricos e meristícos dos exemplares coletados.

Palavras-chave: ocorrência, morfometria, Bahia. 


\section{Introdução}

Segundo Ramos (1998), Catathyridium Chabanaud 1928 é um gênero monofilético, com três espécies reconhecidas: C. garmani, de áreas estuarinas e marinhas costeiras do sudeste do Brasil, Uruguai e Lagoa dos Patos; C. lorentzii (Weynbergh 1895), dos rios Paraguai e Uruguai e C. jenynsi (Günther 1862), dos rios Paraguai/Paraná e Uruguai. Catathyridium grandirivi Chabanaud 1928, descrita do "Rio Grande do Sul e Mar Pequeña", é sinônimo júnior de C. garmani. Entretanto, Eschmeyer (2008) reconhece $C$. grandirivi como válida.

Catathyridium garmani (Jordan \& Goss 1889) (Figura 1) atinge cerca de $20 \mathrm{~cm}$ de comprimento total e sua distribuição previamente

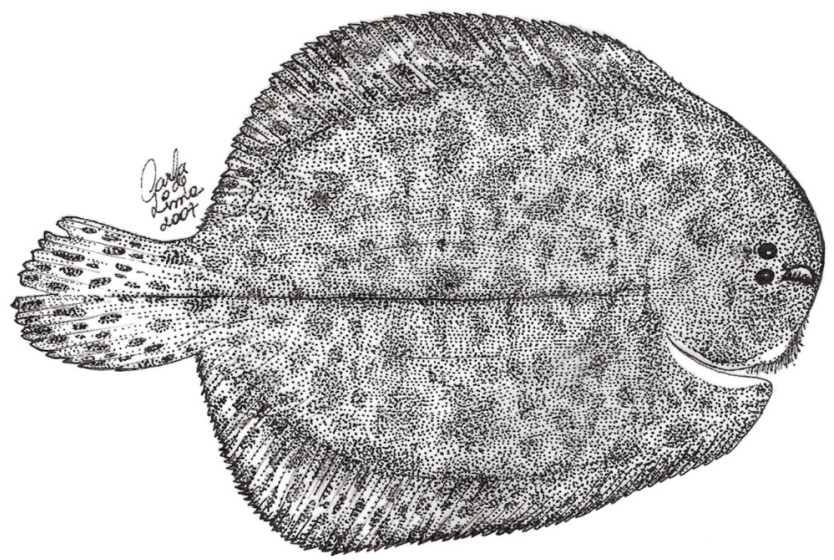

Figura 1. Vista dorsal de Catathyridium garmani, LIUEFS 9411, 68,6 mm comprimento padrão; Estuário do rio Paraguaçu, município de São Félix, Bahia.

Figure 1. Dorsal view of Catathyridium garmani, LIUEFS 9411, $68.6 \mathrm{~mm}$ standart length; Rio Paraguaçu estuary, São Félix, Bahia. conhecida limitava-se das drenagens do estado do Rio de Janeiro (Brasil) ao norte da Argentina (Figueiredo \& Menezes 2000). Habita águas rasas de mangues e estuários, sobre fundos de areia, lodo ou cascalho, preferindo águas de salinidade baixa e podendo atingir água doce (Figueiredo \& Menezes 2000, Fischer et al. 2004, Nelson 2006, Ramos 2003). Os estudos desenvolvidos sobre C. garmani no litoral brasileiro são poucos e estão limitados à filogenia ou levantamentos ictiofaunísticos como, por exemplo, Carvalho et al. (1968), Lema et al. (1980), Chao et al. (1982) e Ramos (1998, 2003).

No presente estudo registra-se pela primeira vez a ocorrência de C. garmani na costa nordeste do Brasil, com base em exemplares coletados no estado da Bahia, ampliando-se os limites de distribuição geográfica da espécie.

\section{Material e Métodos}

O material examinado, distribuído em seis lotes e totalizando 26 exemplares, foi coletado no estuário do rio Paraguaçu, à jusante da Barragem de Pedra do Cavalo, município de São Félix, Bahia (Figura 2), durante os meses de abril e julho de 2005, janeiro de 2006 e julho de 2007, com auxílio de rede de arrasto de fundo. Após a coleta, os exemplares foram fixados em formol $10 \%$ e conservados em álcool 70\%, encontrando-se depositados na coleção científica da Divisão de Peixes do Museu de Zoologia da Universidade Estadual de Feira de Santana, registrado sob os números LIUEFS 6825, 6831, 7044, 7334, 9410 e 9411.

Dados morfométricos foram obtidos com auxílio de paquímetro digital com precisão de $0,05 \mathrm{~mm}$ e foram baseados nas definições propostas por Dawson (1964) e Cervigón et al. (1992). Os dados merísticos e as proporções corporais (diâmetro orbital/comprimento da cabeça e diâmetro orbital/comprimento do focinho) foram comparados com dados de Figueiredo \& Menezes (2000) e as demais pro-

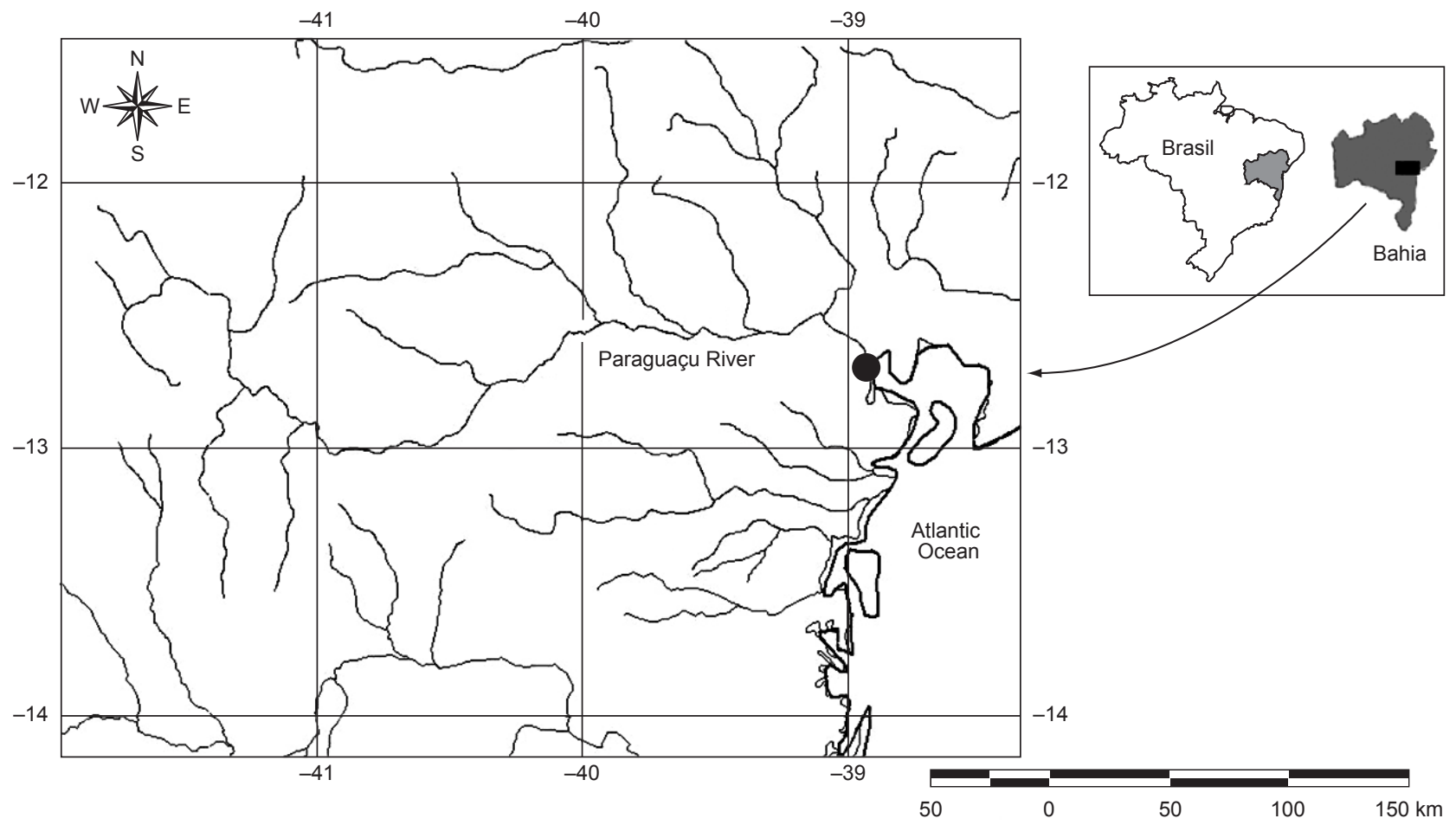

Figura 2. Bacia do rio Paraguaçu, destacando a localidade de coleta no estuário (círculo preto), que representa a ampliação do limite setentrional de distribuição geográfica de Catathyridium garmani.

Figure 2. Rio Paraguaçu Basin, showing the sample site in the estuary (black circle), which represents the ampliation of Northern limit of geographic distribution of Catathyridium garmani. 
porções corporais foram baseadas em Cervigón (1996) para a família Achiridae. A identificação em nível genérico e específico seguiu as diagnoses apresentadas por Figueiredo \& Menezes (2000).

\section{Resultados}

Os comprimentos padrões dos exemplares examinados variaram de 45,24 a 76,98 mm. Os caracteres merísticos e morformétricos bem como as proporções corporais estão apresentados na Tabela 1. A identificação a nível genérico está baseada na presença de um orifício localizado ventralmente no septo interbranquial segundo Figueiredo \& Menezes (2000), sendo a identificação específica também confirmada através deste caráter diagnóstico.

Quanto ao colorido (em álcool 70\%), o lado com olhos apresentase de cor marrom com manchas escuras arredondadas a alongadas, muitas vezes se aglomerando e formando outras de maior tamanho em exemplares de maior porte. Essas manchas também estão presentes nas nadadeiras dorsal, anal e caudal formando faixas interrompidas nas membranas interradiais (exceto na nadadeira caudal). Estrias verticais escuras, em número variável, presentes no corpo. Cirros com pigmentação escura espalhados pelo corpo e em toda linha lateral, que se estende até o final da nadadeira caudal.

\section{Discussão}

No que se refere aos caracteres merísticos, os exemplares de C. garmani examinados diferem das informações citadas por Figueiredo e Menezes (2000) e Fisher et al. (2004) quanto aos números de raios das nadadeiras dorsal (53-58 contra 55-60) e anal (39-44 contra 40-50), segundo Figueiredo e Menezes (2000).

Quanto aos caracteres morfométricos, foram observadas variações no que se refere às proporções diâmetro orbital/comprimento do focinho e diâmetro orbital/comprimento da cabeça (Tabela 1), quando comparado com o que citado por Figueiredo \& Menezes (2000) que citam o olho maior que o espaço interorbital, cabendo 1,5-2,0 vezes no comprimento focinho e 6-7 vezes no comprimento da cabeça. As demais proporções corporais analisadas não foram comparadas devido à ausência de dados disponíveis na literatura.

Tabela 1. Dados merísticos e morformétricos dos 26 exemplares de Catathyridium garmani coletados no estuário do Rio Paraguaçu, São Félix, Bahia (proporções corporais no comprimento padrão são expressos em \%).

Table 1. Meristic and morphometrics data of 26 specimens of Catathyridium garmani collected in Rio Paraguaçu estuary, São Félix, Bahia (body proportions in standard length \%).

\begin{tabular}{lcc}
\hline Caracteres merísticos e morfométricos & $\mathbf{N}=\mathbf{2 6}$ & $\overline{\mathbf{X}}$ \\
\hline Número de raios na nadadeira dorsal & $53-58$ & 54,6 \\
Número de raios da nadadeira anal & $39-42$ & 41,8 \\
Número de raios da nadadeira peitoral & 2 & 2 \\
Número de raios da nadadeira pélvica & 5 & 5 \\
Comprimento total & $63,0-104,0$ & 86,5 \\
Comprimento padrão & $45,2-76,9$ & 64,8 \\
Diâmetro orbital/comprimento da cabeça & $7,5-11,2$ & 9,1 \\
Diâmetro orbital/comprimento do focinho & $2,4-3,9$ & 2,9 \\
Comprimento da cabeça/comprimento & $27,6-33,5$ & 29,9 \\
padrão & & \\
Altura do corpo/comprimento padrão & $57,9-68,1$ & 61,9 \\
Comprimento do focinho/ & $8,3-11,1$ & 9,5 \\
comprimento padrão & & \\
Diâmetro orbital/comprimento padrão & $2,6-3,8$ & 3,3 \\
Diâmetro interorbital/comprimento padrão & $2,6-3,9$ & 3,1 \\
\hline
\end{tabular}

Estas diferenças refletem o pouco conhecimento disponível a respeito da variação intra-específica de $C$. garmani tendo em vista os poucos estudos realizados sobre esta espécie e o pequeno número de exemplares examinados neste estudo. São necessários mais registros de indivíduos em ecossistemas aquáticos locais para confirmar as diferenças morfológicas e merísticas e caracterizar as populações de $C$. garmani nesta região.

O registro da espécie no Rio Paraguaçu, um pouco antes de sua foz na Baía de Todos os Santos, estende os limites de distribuição da espécie e do gênero de $22^{\circ}$ para $13^{\circ}$ de latitude Sul. Deste modo, é ampliado o limite setentrional de distribuição geográfica de $C$. garmani, o qual passa a ser o litoral do estado da Bahia, região nordeste do Brasil.

\section{Agradecimentos}

Os autores agradecem à Carla Lima da UEFS pelo desenho de C. garmani e a Marcelo Brito (UFS) pela confecção do mapa. Alexandre Clistenes é bolsista de Pós-doutorado do CNPq e Perimar Moura recebeu bolsa DTI/CNPq do Programa de Pesquisa em Biodiversidade do Semi-Árido (PPBio).

\section{Referências Bibliográficas}

CARVALHO, J.P., TOMMASI, L.R. \& NOVELLI, M.D. 1968. Lista dos linguados do Brasil. Contrções Inst. Oceanogr. Univ. S. Paulo, sér. Ocean. Biol. (14):1-26.

CERVIGÓN, F., CIPRIANI, R., FISCHER, W., GARIBALDI, L., HENDRICKX, M., LEMUS, A.J., MÁRQUEZ, R., POUTIERS, J.M., ROBAINA, G. \& RODRIGUEZ, B. 1992. Guia de campo de las especies comerciales marinas y de aguas salobres de la costa septentrional de Sur America. Organización de las Naciones Unidas para la Agricultura y la Alimentación, Roma.

CERVIGÓN, F. 1996. Los peces marinos de Venezuela. 2 ed. Fundación Científica los Roques, Caracas.

CHAO, L.N., PEREIRA, L.E., VIEIRA, J.P., BEMVENUTI, M.A. \& Cunha, L.P.R. 1982. Relação preliminar dos peixes estuarinos e marinhos da lagoa dos Patos e região costeira adjacente, Rio Grande do Sul, Brasil. Atlântica, 5(1):67-75.

DAWSON, C.E. 1964. A revision of the Western Atlantic flatfish genus Gymnachirus (the naked soles). Copeia, 1964(4):646-665.

ESCHMEYER, W.N. 2008. Catalog of fishes. Available from: <http://www. calacademy.org/research/ichthyology/catalog/fishcatsearch.html>. (último acesso em 16/10/2008).

FIGUEIREDO, J.L. \& MENEZES, N.A. 2000. Manual de peixes marinhos do sudeste do Brasil. VI. Teleostei (5). Museu de Zoologia da Universidade de São Paulo, São Paulo.

FISCHER, L.G., PEREIRA, L.E.D. \& VIEIRA, J.P. 2004. Peixes estuarinos e costeiros. Ecoscientia, Rio Grande.

LEMA, T., OLIVEIRA, M.F.T. \& LUCENA, C.A.S. 1980. Levantamento preliminar dos Pleuronectiformes do extremo sul do Brasil ao Rio de La Plata (Actinopterygii: Teleostei) Iheringia, Ser. Zool. (56):25-52.

RAMOS, R.T.C. 1998. Estudo filogenético da família Achiridae (Teleostei: Pleuronectiformes: Pleuronectoidei), com a revisão das formas de água doce da América do Sul Cis-Andina e a reavaliação do monofiletismo de soleomorpha (Soleoidei). Tese de Doutorado, Universidade de São Paulo, São Paulo.

RAMOS, R.T.C. 2003. Achiridae (American soles). In Checklist of the freshwater fishes of South and Central America (R.E. Reis, S.O. Kullander \& C.J. Ferraris Jr., eds.). EDIPUCRS, Porto Alegre, p. 666-669.

NELSON, J.S. 2006. Fishes of the world. 4 ed. John Willey \& Sons, New Jersey. 
